\title{
ARTICLE
}

Clinical Study

\section{Oestrogen receptor status and survival in women with BRCA2- associated breast cancer}

\author{
Kelly Metcalfe ${ }^{1,2}$, Henry T. Lynch ${ }^{3}$, William D. Foulkes ${ }^{4}$, Nadine Tung ${ }^{5}$, Olufunmilayo I. Olopade ${ }^{6}$, Andrea Eisen ${ }^{7}$, Jordan Lerner-Ellis ${ }^{8}$, \\ Carrie Snyder $^{3}$, Shana J. Kim ${ }^{1}$, Ping Sun ${ }^{1}$ and Steven A. Narod ${ }^{1}$
}

\begin{abstract}
BACKGROUND: To evaluate the predictors of mortality, including ER status, in women with a BRCA2 mutation and breast cancer. METHODS: Eligible participants were identified from within two longitudinal cohorts. These patients were selected because they were diagnosed with breast cancer between 1975 and 2015 and carried a BRCA2 mutation. Data were abstracted from the medical record and pathology report. We analysed the effects of ER status and other variables on breast cancer specific survival using a Cox proportional hazards model.

RESULTS: Three hundred ninety women with breast cancer and a BRCA2 mutation were included in the analysis. The mean followup time was 12.3 years (range 1-39 years) and 89 subjects died (22.8\%). In the multivariate analysis, women with ER-positive tumours were more likely to die than women with ER-negative tumours ( $\mathrm{HR} 2.08,95 \% \mathrm{Cl} 0.99-4.36, p=0.05$ ), and this was of borderline significance. For the 233 women with ER-positive tumours the 20 -year survival rate was $62.2 \%$, compared to $83.7 \%$ for 58 women with ER-negative tumours $(p=0.03)$.

CONCLUSIONS: The majority of women with a BRCA2 mutation present with ER-positive breast cancer, and for these women, prognosis may be worse than for BRCA2 carriers with ER-negative breast cancer.
\end{abstract}

British Journal of Cancer (2019) 120:398-403; https://doi.org/10.1038/s41416-019-0376-y

\section{INTRODUCTION}

Women with a mutation in BRCA2 have a lifetime risk of developing breast cancer of $\sim 70 \%{ }^{1}$ Approximately two percent of all breast cancers are due to mutations in the BRCA2 gene, and there is emerging evidence that women with a BRCA2 mutation have a worse prognosis compared to women with a BRCA1 mutation or with a sporadic breast cancer. ${ }^{2,3}$ This difference could be attributed to the fact that BRCA2 mutation carriers present with more aggressive tumours compared to women without a mutation, and BRCA2 oestrogen receptor (ER)-positive breast cancers tend to be luminal $B^{4}$ and have higher oncotype recurrence scores compared to sporadic breast cancers. $^{5-7}$ Alternatively, the BRCA2 mutation could be an independent predictor beyond the pathological characteristics of the cancer.

Women with sporadic breast cancer experience better survival if they present with ER-positive tumours, compared to women with ER-negative tumours. ${ }^{8,9}$ However, it has recently been reported that the opposite relationship is observed in women with BRCA2associated breast cancers. Jonasson et al. reported that among 285 women with a founder Icelandic BRCA2 mutation, positive ER-status was associated with a greater risk of death, compared to negative ER status. ${ }^{10}$ In that study, after adjustment for other prognostic factors and treatments, women with a BRCA2 mutation had worse long-term survival compared to women without a BRCA2 mutation; this difference was mainly observed in women with ER-positive breast cancer. Their study only included women with a specific BRCA2 mutation (999del5) and it is unclear if the same pattern is observed in women with other BRCA2 mutations. In the current study, we report on the predictors of mortality, including ER status, in women with a BRCA2 mutation and breast cancer.

\section{METHODS}

Eligible subjects were identified through merging of two existing cohorts of women with breast cancer and a BRCA2 mutation, including a North American BRCA-associated breast cancer treatment study cohort ${ }^{11}$ and an international BRCA risk factor study. ${ }^{12}$ The study sample for the North American cohort study included women with a BRCA2 mutation diagnosed with stage I or stage II breast cancer at age 65 or below, between 1975 and 2008. The international risk factor study includes women with a $B R C A$ mutation from 35 centres in nine countries who are followed prospectively from time of genetic testing. These cases included prevalent cases at the time of enrolment and incident cases diagnosed in the follow-up period among women who were cancer free at the time of enrolment.

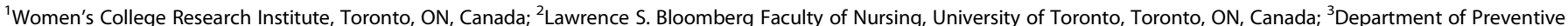

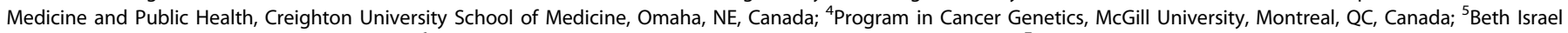

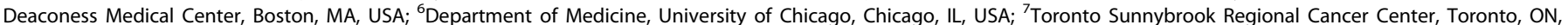
Canada and ${ }^{8}$ Pathology and Laboratory Medicine, Mount Sinai Hospital, Toronto, ON, Canada

Correspondence: Steven A. Narod (steven.narod@wchospital.ca)

Received: 23 May 2018 Revised: 6 December 2018 Accepted: 3 January 2019

Published online: 6 February 2019 
Table 1. Frequency and mean values for related variables of the 390 subjects with BRCA2 mutations

\begin{tabular}{ll}
\hline Variables & Mean or frequency \\
\hline Date of birth & $1950.1(1899-1978)$ \\
Date of diagnoses (range) & $1996.1(1975-2015)$ \\
$1975-1980$ & $32(8.2 \%)$ \\
$1981-1990$ & $77(19.7 \%)$ \\
$1991-2000$ & $159(40.8 \%)$ \\
$2001-2010$ & $102(26.2 \%)$ \\
$2010-2015$ & $20(5.1 \%)$ \\
Age at diagnoses (range) & $46.0(24-103)$ \\
Years of follow-up (mean) (range) & $12.3(0-39)$ \\
Vital status & \\
Alive & $236(60.5 \%)$ \\
Dead & $89(22.8 \%)$ \\
Lost to follow-up & $65(16.7 \%)$ \\
Cause of death & \\
Breast cancer & 60 \\
Other & 16 \\
Missing & 13 \\
\hline
\end{tabular}

All study procedures were approved by the institutional review boards at each of the participating centres. For the current study, we included only subjects with BRCA2 mutation who were diagnosed with invasive breast cancer between 1975 and 2015, and for whom a pathology report or treatment record was available. We identified a total of 390 eligible subjects, including 315 from the North American cohort study, and 75 from the international prospective cohort study.

\section{Study protocol}

The treatment records and pathology documents were reviewed. We recorded tumour size (in centimeters), nodal status (positive/ negative) and tumour grade (I-III). ER status was recorded as positive, negative, equivocal or unknown. We recorded the use of chemotherapy (yes/no), tamoxifen (yes/no), radiotherapy (yes/no) and bilateral salpingo-oophorectomy (yes/no). Vital status was recorded at time of last follow-up as living or deceased. Vital status was provided by the clinician/investigator affiliated with the centre for the subject by review of hospital medical records and in some cases communication with the next of kin.

Statistical analysis

A series of survival analyses were performed. The primary endpoint was death. We chose this endpoint because the cause of death was missing in 13 of the 89 women who died. We considered the woman to be at risk for death from the date of the first surgical procedure until the last date of follow-up or until death. Hazard ratios were estimated using the Cox proportional hazards model, implemented in SAS. We evaluated the use of tamoxifen and chemotherapy as dichotomous variables. Oophorectomy was evaluated as a time-dependent variable. We estimated the effect of ER status in the entire patient population and then in subgroups defined by age at diagnosis $(<50$ or $>50)$. The hazard ratios were adjusted for age of diagnosis, tumour size, lymph node status, chemotherapy, tamoxifen and oophorectomy.

\section{RESULTS}

The characteristics of the 390 subjects with BRCA2-associated breast cancer are presented in Table 1. The women were diagnosed between 1975 and 2015 and were followed for a mean of 12.3 years (range 1-39 years).

During the follow-up period, 89 subjects died (22.8\%). Of these 60 died from breast cancer and the cause of death was missing for 13 patients. The ten-year breast cancer survival rate for the entire cohort was $83.3 \%$.

Table 2 presents the relative risks of death according to demographic, clinical and treatment variables. The variable that most strongly predicted mortality in women with BRCA2associated breast cancer was positive ER status; in the multivariate analysis, women with ER-positive tumours were more likely to die than women with ER-negative tumours (HR 2.08, 95\% Cl 0.99-4.36, $p=0.05)$. Two hundred thirty-three women had ER-positive tumours; for them, the 20 -year survival rate was $62.2 \%$, compared to $83.7 \%$ in women with ER-negative tumours $(p=0.03)$ (Fig. 1). For women under age 50 at diagnosis (Fig. 2), the survival disadvantage associated with ER-positive tumours as compared to ER-negative tumours was even more profound (20-year survival rate 68.3 vs. $91.3 \% ; p=0.02$ ).

Women with ER-negative breast cancers were significantly more likely to have grade III tumours than women with ER-positive breast cancers $(p<0.001)$. There were no significant differences in tumour size $(p=0.66)$ or nodal status $(p=0.16)$ between women with ER-negative breast cancer compared to ER-positive breast cancer.

Table 3 presents the relative risks of mortality for BRCA2 carriers with ER-positive tumours associated with various treatment variables (including chemotherapy, radiation therapy and tamoxifen). In the multivariate analysis, there was no observed reduction in mortality in BRCA2 mutation carriers with ER-positive breast tumours associated with the use of tamoxifen $(\mathrm{HR}=0.91 ; 95 \%$ $\mathrm{Cl} 0.49-1.69, p=0.76)$ (Fig. 3a) or with chemotherapy (HR=1.03; $95 \% \mathrm{Cl} 0.51-2.06, p=0.94$ ) (Fig. 3b).

\section{DISCUSSION}

In this international cohort study of 390 breast cancer patients with a BRCA2 mutation, positive ER status was associated with a relatively poor prognosis compared to ER-negative status. Women with ER-positive tumours had a ten-year survival rate of $80.4 \%$, compared to $92.6 \%$ in women with ER-negative tumours. After controlling for pathologic features and cancer treatments, positive ER status was the strongest predictor of mortality in this cohort of women with BRCA2-associated breast cancer $(\mathrm{HR}=2.08 ; 95 \% \mathrm{Cl}$ 0.99-4.36, $p=0.05$ ). Furthermore, in women with ER-positive tumours, the use of tamoxifen or chemotherapy did not appear to improve survival.

We hereby confirm the findings of Jonasson et al. (2016) who reported that among Icelandic BRCA2 carriers, ER-positive status was an adverse prognostic factor. ${ }^{10}$ Their study included 285 breast cancer patients with a 999del5 BRCA2 mutation, matched with 570 non-carrier patients. After adjustment for various prognostic factors and treatments, a BRCA2 mutation was associated with a significantly worse prognosis than a sporadic breast cancer $(\mathrm{HR}=1.61 ; 95 \% \mathrm{Cl} 1.11-2.35, p=0.01)$. However, this worse prognosis was only observed among women with ERpositive tumours $(\mathrm{HR}=1.92,95 \% \mathrm{Cl} 1.20-3.05, p=0.006)$, and not for women with ER-negative tumours $(H R=1.12,95 \%$ $\mathrm{Cl} 0.54-2.31, p=0.77$ ).

The majority of breast cancers diagnosed in women with a BRCA2 mutation are ER-positive. ${ }^{2,13}$ BRCA2 tumours are significantly more likely to be ER-positive compared to both BRCA1 tumours and sporadic tumours. ${ }^{13}$ We observed this in the current cohort, with $77 \%$ of the BRCA2 carriers having ER-positive tumours.

Breast cancer prognostic factors typically include tumour size, nodal status and grade. In the current study of women with BRCA2 mutations, there were no significant differences in mortality 
Table 2. Relative risk (RR) on death for related variables (all subjects)

\begin{tabular}{|c|c|c|c|c|}
\hline Variables & $\mathrm{N}$ & $\begin{array}{l}\mathrm{N} \text { deaths percent } \\
\text { read by row }\end{array}$ & Univariate RR $(95 \% \mathrm{Cl}), \mathrm{P}$ & Multivariate RR $(95 \% \mathrm{Cl}) \mathrm{P}$ \\
\hline Age at diagnosis (years) & 390 & 89 (22.8\%) & $1.02(0.99-1.04), 0.15$ & $1.02(0.99-1.04), 0.23$ \\
\hline Negative & $58(14.9 \%)$ & $9(15.5 \%)$ & 1 & 1 \\
\hline Positive & $233(59.7 \%)$ & $51(21.9 \%)$ & $1.94(0.95-3.95), 0.07$ & $2.08(0.99-4.36), 0.05$ \\
\hline Borderline & $9(2.3 \%)$ & 0 & & \\
\hline \multicolumn{5}{|l|}{ Mastectomy } \\
\hline No & 169 (43.3\%) & 33 (19.5\%) & 1 & 1 \\
\hline Yes & $221(56.7 \%)$ & $56(25.3 \%)$ & $1.13(0.73-1.74), 0.59$ & $1.38(0.68-2.79), 0.37$ \\
\hline \multicolumn{5}{|l|}{ Grade } \\
\hline 1 & $23(5.9 \%)$ & $3(13.0 \%)$ & 1 & 1 \\
\hline Negative & $203(52.1 \%)$ & 40 (19.7\%) & 1 & 1 \\
\hline Positive & $145(37.2 \%)$ & 39 (26.9\%) & $1.36(0.87-2.11), 0.18$ & $0.98(0.55-1.77), 0.95$ \\
\hline Missing & $42(10.8 \%)$ & $10(23.8 \%)$ & & \\
\hline \multicolumn{5}{|l|}{ Size $(\mathrm{mm})$} \\
\hline $0-10$ & 87 (22.3\%) & 13 (14.9\%) & 1 & 1 \\
\hline $11-20$ & $143(36.7 \%)$ & $36(25.2 \%)$ & $1.37(0.73-2.59), 0.33$ & $1.65(0.84-3.23), 0.15$ \\
\hline $21-30$ & $76(19.5 \%)$ & $22(29.0 \%)$ & $1.67(0.84-3.31), 0.14$ & $1.84(0.85-3.95), 0.12$ \\
\hline $31+$ & $61(15.6 \%)$ & 15 (24.6\%) & $1.94(0.92-4.08), 0.08$ & $2.21(0.95-5.17), 0.07$ \\
\hline Missing & $23(5.9 \%)$ & $3(13.0 \%)$ & & \\
\hline \multicolumn{5}{|l|}{ Oophorectomy ${ }^{a}$} \\
\hline \multicolumn{5}{|l|}{ Radiation therapy } \\
\hline No & 195 (50\%) & $47(24.1 \%)$ & 1 & 1 \\
\hline Yes & $167(42.8 \%)$ & $36(21.6 \%)$ & $0.94(0.61-1.45), 0.76$ & $1.38(0.69-2.73), 0.36$ \\
\hline Missing & $28(7.2 \%)$ & $6(21.4 \%)$ & & \\
\hline \multicolumn{5}{|l|}{ Chemotherapy } \\
\hline No & 141 (36.2\%) & 37 (26.2\%) & 1 & 1 \\
\hline Yes & $221(56.7 \%)$ & $46(20.8 \%)$ & $1.05(0.68-1.63), 0.83$ & $1.00(0.57-1.74), 1.00$ \\
\hline Missing & $28(7.2 \%)$ & $6(21.4 \%)$ & & \\
\hline
\end{tabular}

associated with these factors. In the adjusted model, mortality was not associated with high grade tumours $(\mathrm{HR}=0.77 ; 95 \%$ $\mathrm{Cl} 0.21-2.80, p=0.69$ ), or with positive lymph node status $(\mathrm{HR}=0.98 ; 95 \% \mathrm{Cl} 0.55-1.77, p=0.95)$. The sample size was relatively small and the confidence limits are wide, and it is important that these results be confirmed in other settings.

ER-status has been shown to be an independent favourable predictor of outcome in women with breast cancer. ${ }^{14}$ However, certain subgroups of patients with breast cancer do not experience a favourable prognosis associated with positive ER status, including women diagnosed with breast cancer under the age of 40 years. In a recent analysis of 1910 Canadian women with breast cancer, the prognostic effect of ER-status differed according to age of diagnosis. ${ }^{15}$ Among 213 women diagnosed with breast cancer under the age of 40 years, 15 -year survival was significantly worse for women with ER-positive tumours, compared to those with ER-negative tumours (55 vs. $61 \%$ ). For those diagnosed with breast cancer over the age of 40 years, positive ER status was a favourable prognostic factor.

We have previously reported that oophorectomy reduces the risk of long-term mortality in women with BRCA-associated breast cancer by 54\%; however, this benefit was significant for women with a BRCA1 mutation ( $(\mathrm{HR}=0.38 ; 95 \% \mathrm{Cl} 0.19-0.77, p=0.007)$, but was not significant for women with a BRCA2 mutation 

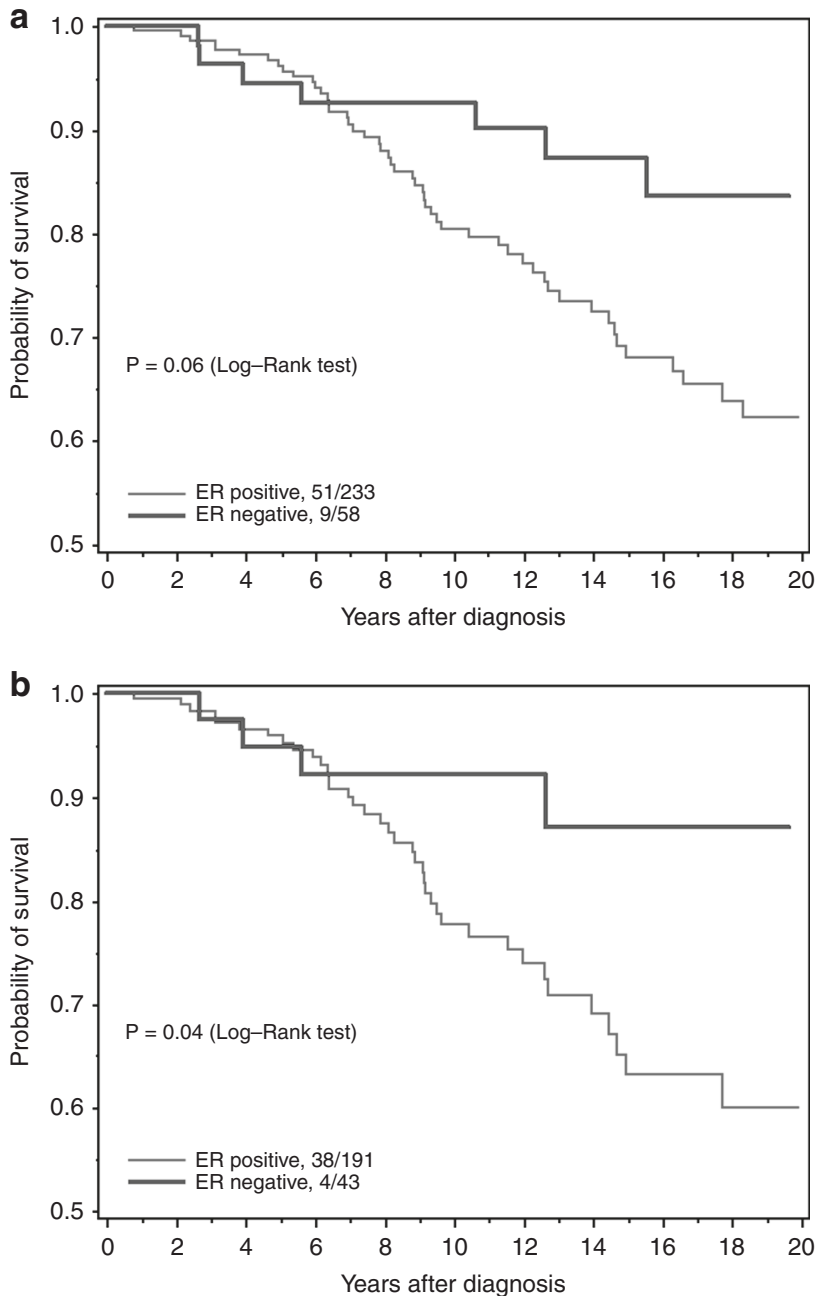

Fig. 1 a All-cause survival by ER status. b All-cause survival for women diagnosed after 1990, by ER status

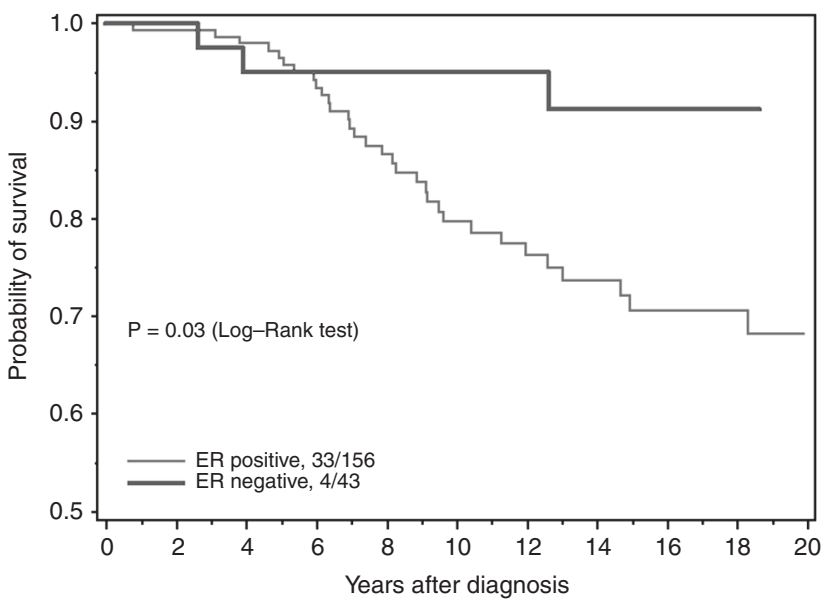

Fig. 2 All-cause survival for women under 50, by ER status

( $\mathrm{HR}=0.57,95 \% \mathrm{Cl} 0.23-1.43, p=0.23)$, although the latter group was small. ${ }^{11}$ Furthermore, the beneficial effect of oophorectomy was only observed in women with ER-negative breast cancers $(\mathrm{HR}=0.07 ; 95 \% \mathrm{Cl} 0.01-0.51, p=0.009)$, and not for women with ER-positive breast cancers ( $\mathrm{HR}=0.76 ; 95 \% \mathrm{Cl} 0.32-1.78, p=0.53$ ). In the current study, we also did not observe a survival benefit

\begin{tabular}{|c|c|c|}
\hline Variables & $\begin{array}{l}\text { Univariate RR }(95 \% \\
\mathrm{Cl}), \mathrm{P}\end{array}$ & $\begin{array}{l}\text { Multivariate RR }(95 \% \\
\mathrm{Cl}), \mathrm{P}\end{array}$ \\
\hline Age at diagnosis & $1.00(0.97-1.03), 1.00$ & $1.00(0.97-1.04), 0.92$ \\
\hline \multicolumn{3}{|l|}{ Grade } \\
\hline | or II & 1 & 1 \\
\hline III & $0.57(0.25-1.31), 0.19$ & $0.48(0.20-1.16), 0.10$ \\
\hline \multicolumn{3}{|l|}{ Nodal status } \\
\hline Negative & 1 & 1 \\
\hline Positive & $1.24(0.71-2.18), 0.45$ & $1.02(0.48-2.14), 0.97$ \\
\hline \multicolumn{3}{|l|}{ Size $(\mathrm{mm})$} \\
\hline $0-10$ & 1 & 1 \\
\hline $11-20$ & $1.33(0.62-2.88), 0.46$ & $1.65(0.84-3.23), 0.15$ \\
\hline $21-30$ & $1.41(0.59-3.37), 0.43$ & $1.84(0.85-3.95), 0.12$ \\
\hline $31+$ & $1.04(0.37-2.93), 0.94$ & $2.21(0.95-5.17), 0.07$ \\
\hline \multicolumn{3}{|l|}{ Mastectomy } \\
\hline No & 1 & 1 \\
\hline Yes & $1.05(0.61-1.83), 0.86$ & $1.27(0.97-1.04), 0.92$ \\
\hline \multicolumn{3}{|l|}{ Tamoxifen } \\
\hline No & 1 & 1 \\
\hline Yes & $0.90(0.51-1.58), 0.72$ & $0.91(0.49-1.69), 0.76$ \\
\hline \multicolumn{3}{|l|}{ Radiation therapy } \\
\hline No & 1 & 1 \\
\hline Yes & $1.12(0.63-1.97), 0.70$ & $1.41(0.56-3.50), 0.46$ \\
\hline \multicolumn{3}{|l|}{ Chemotherapy } \\
\hline No & 1 & 1 \\
\hline Yes & $0.99(0.55-1.77), 0.98$ & $1.03(0.51-2.06), 0.94$ \\
\hline $\begin{array}{c}\text { For subjects } \\
\text { diagnosed after } 1990\end{array}$ & $1.15(0.57-2.33), 0.70$ & $1.08(0.48-2.43), 0.85$ \\
\hline \multicolumn{3}{|l|}{ Oophorectomy ${ }^{a}$} \\
\hline No & 1 & 1 \\
\hline Yes & $1.10(0.83-1.45), 0.51$ & $1.18(0.86-1.62), 0.30$ \\
\hline
\end{tabular}

associated with oophorectomy in women with BRCA2-associated breast cancer.

Adjuvant hormone therapy (tamoxifen) is indicated for women with ER-positive tumours, and has been shown to decrease the risk of local recurrence and death. ${ }^{16-18}$ However, there is evidence from several studies that a beneficial effect of adjuvant hormone therapy is not observed in women with BRCA2associated breast cancer. Goodwin et al. reported that for breast cancer patients that had taken adjuvant hormone therapy, women with a BRCA2 mutation had a higher risk of death compared to sporadic breast cancers (HR $=2.05 ; 95 \% \mathrm{Cl} 1.07-3.91 ; p=0.03){ }^{2}$ In the current study, the use of tamoxifen did not significantly decrease the risk of death in women with ER-positive breast cancer ( $\mathrm{HR}=0.91 ; 95 \% \mathrm{Cl} 0.49-1.69, p=0.76)$, in support of the findings of Jonasson et al. (2016) ( $\mathrm{HR}=1.03 ; 95 \% \mathrm{Cl} 0.41-2.60)$.

In 285 Icelandic women with BRCA2 mutations, the use of adjuvant chemotherapy decreased the risk of mortality $(\mathrm{HR}=0.35$; $95 \% \mathrm{Cl} 0.16-0.80, p=0.01$ ); this was not observed in (matched) sporadic breast cancers in Iceland $(\mathrm{HR}=0.98 ; 95 \% \mathrm{Cl} 0.47-2.04$, $p=0.96) .{ }^{10}$ In the current study, we observe a significant benefit associated with chemotherapy neither in women with BRCA2associated breast cancer ( $\mathrm{HR}=1.00 ; 95 \% \mathrm{Cl} 0.57-1.74, p=1.00)$, nor in the subgroup of women with ER-positive tumours $(\mathrm{HR}=1.03 ; 95 \% \mathrm{Cl} 0.51-2.06 ; \mathrm{p}=0.94)$. More research is needed 

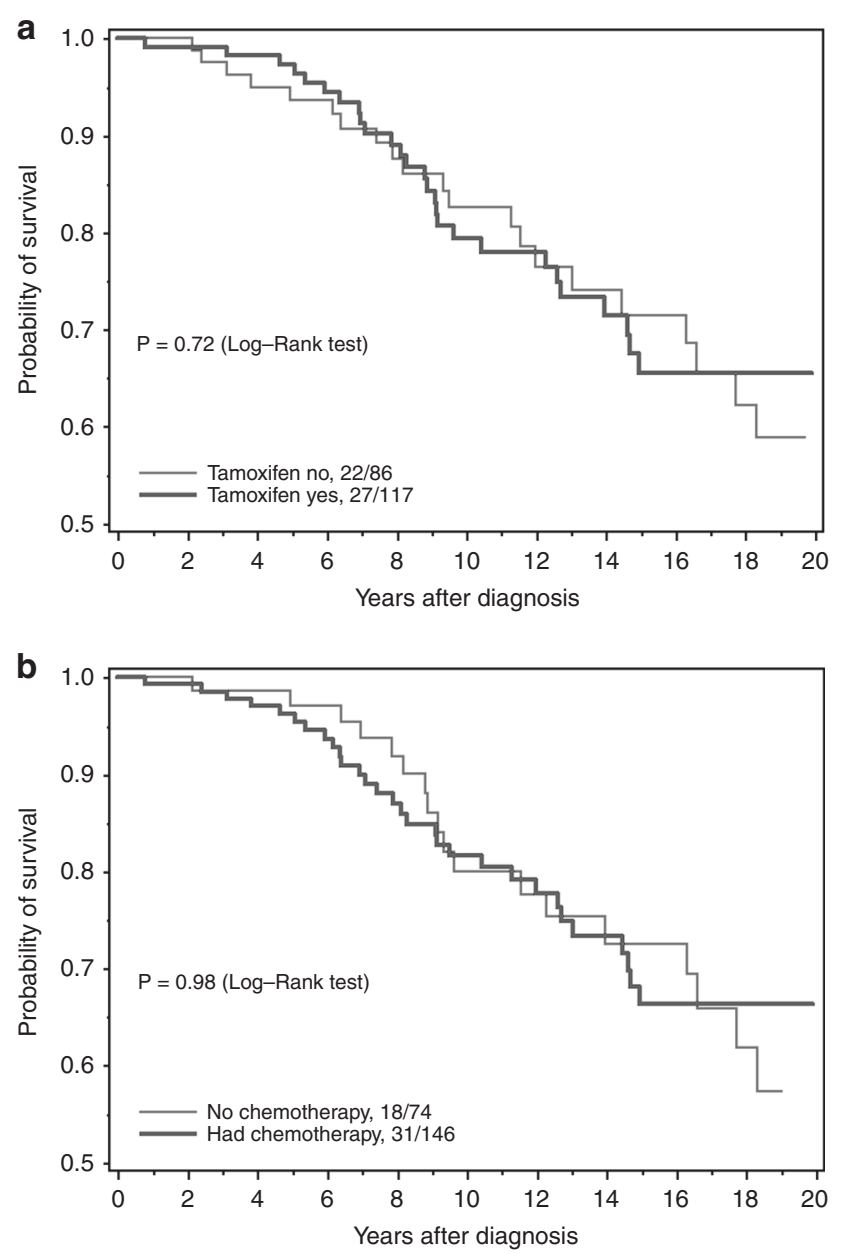

Fig. 3 a All-cause survival among ER-positive breast cancer, by tamoxifen. b All-cause survival among ER-positive breast cancer, by chemotherapy use

to evaluate the effect of various chemotherapy regimens on survival in women with BRCA2-associated breast cancer, in particular with platinum-based regimens, which have shown preliminary evidence of effectiveness in BRCA1 mutation carriers. $^{19-22}$

The POSH study ${ }^{23}$ followed 137 women with a BRCA2 mutation for death and other clinical outcomes, but there were only 21 ER-negative cases among these. They found that BRCA2-positive status did not negatively impact upon survival, but they were unable to evaluate the effect of ER-status on prognosis on the BRCA2-positive subgroup.

There are several limitations to our study. This is a historical cohort study, so participants were not randomised to various treatments. We did not have the date of genetic testing of these women, and therefore we could not exclude the possibility of survivorship bias (this would occur in study subjects overrepresented by long-term survivors); however, there is no reason that this would be different for ER-positive vs. ER-negative cases.

The study was not designed to investigate the effect of various treatments, and it is possible that the women with and without chemotherapy had different risk profiles. Furthermore, women included in this study were diagnosed with breast cancer over a 40-year period (between 1975 and 2015) and treatments have evolved since then, which may have impacted survival estimates. In particular both tamoxifen and chemotherapy were introduced in this timeframe. We do not have the details of the individual chemotherapies but few, if any, patients would have received a platinum-based therapy. ER status was missing in $\sim 20 \%$ of the sample, which may reflect the breast cancer diagnoses years, as ER status was not performed as routinely in the 1970s and early 1980s. The cause of death was missing for 13 patients and therefore we chose all-cause mortality as the principal endpoint. However, given the rarity of BRCA2-positive breast cancers $(\sim 2 \%$ of all breast cancers) it is difficult to construct a large contemporary cohort.

This study adds to the growing evidence of the poor prognosis associated with a BRCA2 mutation and ER-positive breast cancer. The typical breast cancer prognostic factors, including grade, tumour size and nodal status, may not be typical for women with BRCA2 mutations. The observed lack of effective treatments for ERpositive BRCA2-associated breast cancer requires attention, and future research needs to be conducted to evaluate the contribution of newer treatments, including platinum-based chemotherapy, oophorectomy and PARP inhibitors. Moreover, women with a BRCA2 mutation without breast cancer should be counselled on the poor prognosis associated with BRCA2-associated breast cancer when making cancer risk reduction decisions.

\section{FUNDING}

This research was funded by the Canadian Breast Cancer Foundation (Ontario Chapter).

\section{AUTHOR CONTRIBUTIONS}

K.M. and S.A.N obtained funding, conceived and designed the study. P.S. conducted the data analysis. All authors were involved in interpretation of data and manuscript preparation.

\section{ADDITIONAL INFORMATION}

Competing interests: The authors declare no competing interests.

Data availability: K.M. and S.N. had full access to all of the data in the study and take responsibility for the integrity of the data and the accuracy of the data analysis. Data requests may be submitted to K.M., which will be submitted for ethical approval (kelly.metcalfe@utoronto.ca).

Ethical approval: This study was approved by the Women's College Research Ethics Board. All procedures performed in studies involving human participants were in accordance with the ethical standards of the institutional and/or national research committee, and with the 1964 Helsinki declaration and its later amendments or comparable ethical standards.

Informed consent: Informed consent was obtained from all individual participants included in the study.

Note: This work is published under the standard license to publish agreement. After 12 months the work will become freely available and the license terms will switch to a Creative Commons Attribution 4.0 International (CC BY 4.0).

Publisher's note: Springer Nature remains neutral with regard to jurisdictional claims in published maps and institutional affiliations.

\section{REFERENCES}

1. Kuchenbaecker, K. B. et al. Risks of breast, ovarian, and contralateral breast cancer for BRCA1 and BRCA2 mutation carriers. JAMA 317, 2402-2416 (2017).

2. Goodwin, P. J. et al. Breast cancer prognosis in BRCA1 and BRCA2 mutation carriers: an International Prospective Breast Cancer Family Registry populationbased cohort study. J. Clin. Oncol. 30, 19-26 (2012).

3. Rennert, G. et al. Clinical outcomes of breast cancer in carriers of BRCA1 and BRCA2 mutations. N. Engl. J. Med. 357, 115-123 (2007).

4. Mavaddat, N. et al. Pathology of breast and ovarian cancers among BRCA1 and BRCA2 mutation carriers: results from the Consortium Of Investigators of Modifiers of BRCA1/2 (CIMBA). Cancer Epidemiol. Biomark. Prev. 21, 134-147 (2012). 
5. Lewin, R. et al. Oncotype-DX recurrence score distribution in breast cancer patients with BRCA1/2 mutations. Breast Cancer Res. Treat. 157, 511-516 (2016).

6. Halpern, $N$. et al. Oncotype Dx recurrence score among BRCA1/2 germline mutation carriers with hormone receptors positive breast cancer. Int. J. Cancer 140, 2145-2149 (2017).

7. Shah, P. D. et al. Twenty-one-gene recurrence score assay in BRCA-associated versus sporadic breast cancers: differences based on germline mutation status. Cancer 122, 1178-1184 (2016).

8. Parl, F. F., Schmidt, B. P., Dupont, W. D. \& Wagner, R. K. Prognostic significance of estrogen receptor status in breast cancer in relation to tumor stage, axillary node metastasis, and histopathologic grading. Cancer 54, 2237-2242 (1984).

9. Crowe, J. P. et al. Estrogen receptor determination and long term survival of patients with carcinoma of the breast. Surg. Gynecol. Obstet. 173, 273-278 (1991).

10. Jonasson, J. G. et al. Oestrogen receptor status, treatment and breast cancer prognosis in Icelandic BRCA2 mutation carriers. Br. J. Cancer 115, 776-783 (2016).

11. Metcalfe, K. et al. Effect of oophorectomy on survival after breast cancer in BRCA1 and BRCA2 mutation carriers. JAMA Oncol. 1, 306-313 (2015).

12. Kotsopoulos, J. et al. Bilateral oophorectomy and breast cancer risk in BRCA1 and BRCA2 mutation carriers. J. Natl. Cancer Inst. 109, djw177 (2017).

13. Brekelmans, C. T. et al. Tumour characteristics, survival and prognostic factors of hereditary breast cancer from BRCA2-, BRCA1- and non-BRCA1/2 families as compared to sporadic breast cancer cases. Eur. J. Cancer 43, 867-876 (2007).

14. Grann, V. R. et al. Hormone receptor status and survival in a population-based cohort of patients with breast carcinoma. Cancer 103, 2241-2251 (2005).
15. Sopik, V., Sun, P. \& Narod, S. A. The prognostic effect of estrogen receptor status differs for younger versus older breast cancer patients. Breast Cancer Res. Treat. 165, 391-402 (2017).

16. Fisher, B. et al. Tamoxifen for prevention of breast cancer: report of the National Surgical Adjuvant Breast and Bowel Project P-1 study. J. Natl. Cancer Inst. 90, 1371-1388 (1998).

17. Ekholm, M. et al. Two years of adjuvant tamoxifen provides a survival benefit compared with no systemic treatment in premenopausal patients with primary breast cancer: long-term follow-up (>25 years) of the phase III SBII:2pre trial. J. Clin. Oncol. 34, 2232-2238 (2016).

18. Early Breast Cancer Trialists' Collaborative G, Davies, C. et al. Relevance of breast cancer hormone receptors and other factors to the efficacy of adjuvant tamoxifen: patient-level meta-analysis of randomised trials. Lancet 378, 771-784 (2011).

19. Byrski, T. et al. Results of a phase II open-label, non-randomized trial of cisplatin chemotherapy in patients with BRCA1-positive metastatic breast cancer. Breast Cancer Res. 14, R110 (2012).

20. Byrski, T. et al. Pathologic complete response rates in young women with BRCA1positive breast cancers after neoadjuvant chemotherapy. J. Clin. Oncol. 28, 375-379 (2010).

21. Byrski, T. et al. Response to neoadjuvant therapy with cisplatin in BRCA1-positive breast cancer patients. Breast Cancer Res. Treat. 115, 359-363 (2009).

22. Byrski, T. et al. Pathologic complete response to neoadjuvant cisplatin in BRCA1-positive breast cancer patients. Breast Cancer Res. Treat. 147, 401-405 (2014).

23. Copson, E. R. et al. Germline BRCA mutation and outcome in young-onset breast cancer (POSH): a prospective cohort study. Lancet Oncol. 19, 169-180 (2018). 